\title{
Tailored Activation of Middle-Aged Men to Promote Discussion of Recent Active Suicide Thoughts: a Randomized Controlled Trial
}

\author{
Anthony Jerant, $M D^{7}$, Paul Duberstein, $P h D^{2}$, Richard L. Kravitz, MD, $M S P H^{3}$, \\ Deborah M. Stone, SCD, MSW, MPH' ${ }^{4}$, Camille Cipri, BS ${ }^{5}$, and Peter Franks, $\mathrm{MD}^{7}$
}

\begin{abstract}
'Department of Family and Community Medicine, University of California Davis (UCD) School of Medicine, 4860 Y Street, Suite 2300, Sacramento, CA, USA; ${ }^{2}$ Department of Health Behavior, Society, and Policy, Rutgers School of Public Health, 683 Hoes Lane West, Piscataway, NJ, USA; ${ }^{3}$ Division of General Medicine, Department of Internal Medicine, UCD School of Medicine, 4150 V Street, Suite 2400, PSSB, Sacramento, CA, USA; ${ }^{4}$ Division of Violence Prevention, National Center for Injury Prevention and Control, Centers for Disease Control and Prevention, 4770 Buford Highway NE, Atlanta, GA, USA; ${ }^{5}$ Center for Healthcare Policy and Research, UCD, 2103 Stockton Blvd, Sacramento, CA, USA.
\end{abstract}

PURPOSE: Middle-aged men are at high risk of suicide. While about half of those who kill themselves visit a primary care clinician (PCC) shortly before death, in current practice, few spontaneously disclose their thoughts of suicide during the visits, and PCCs seldom inquire about such thoughts. In a randomized controlled trial, we examined the effect of a tailored interactive computer program designed to encourage middle-aged men's discussion of suicide with PCCs.

METHODS: We recruited men 35-74 years old reporting recent (within 4 weeks) active suicide thoughts from the panels of 42 PCCs (the unit of randomization) in eight offices within a single California health system. In the office before a visit, men viewed the intervention corresponding to their PCC's random group assignment: Men and Providers Preventing Suicide (MAPS) (20 PCCs), providing tailored multimedia promoting discussion of suicide thoughts, or control (22 PCCs), composed of a sleep hygiene video plus brief non-tailored text encouraging discussion of suicide thoughts. Logistic regressions, adjusting for patient nesting within physicians, examined MAPS' effect on patient-reported suicide discussion in the subsequent office visit.

RESULTS: Sixteen of the randomized PCCs had no patients enroll in the trial. From the panels of the remaining 26 PCCs (12 MAPS, 14 control), 48 men (MAPS 21, control 27) were enrolled (a mean of 1.8 (range 1-5) per PCC), with a mean age of 55.9 years (SD 11.4). Suicide discussion was more likely among MAPS patients (15/21 [65\%]) than controls (8/27 [35\%]). Logistic regression showed men viewing MAPS were more likely than controls to discuss suicide with their PCC (OR 5.91, 95\% CI 1.5921.94; $P=0.008$; nesting-adjusted predicted effect $71 \%$ vs. $30 \%)$.

CONCLUSIONS: In addressing barriers to discussing suicide, the tailored MAPS program activated middle-aged men with active suicide thoughts to engage with PCCs around this customarily taboo topic.

Received September 20, 2019

Accepted March 4, 2020

Published online March 17, 2020
J Gen Intern Med 35(7):2050-8

DOI: $10.1007 / \mathrm{s} 11606-020-05769-3$

(C) Society of General Internal Medicine 2020

$\mathrm{T}$ here are almost 50,000 suicide deaths in the USA annually, nearly $80 \%$ occurring in men. ${ }^{1}$ Suicide is particularly common among 35-74-year-old (hereafter, middle-aged) men, ${ }^{1,2}$ representing the eighth leading cause of death in this group, ahead of septicemia and other common conditions. ${ }^{2-5}$ Suicide deaths among middle-aged men entail tremendous human and economic costs. ${ }^{4}$

About half of all adults who die by suicide see a primary care clinician (PCC) in the month before death; about 20\% see a mental health specialist. ${ }^{6}$ Yet, nearly all prior clinical suicide prevention efforts have been targeted to patients in specialty settings, typically following a suicide attempt. ${ }^{7-10}$ Such approaches have value but cannot prevent the two thirds of male suicide deaths occurring on first attempt. ${ }^{11,12}$ Public health strategies such as limiting access to lethal means among people at risk have broader reach, ${ }^{13}$ but suicide risk is fourfold higher in clinical samples, ${ }^{14}$ suggesting the potential value of prevention in primary care. ${ }^{15,16}$

Currently, the topic of suicide is rarely broached in primary care visits made by suicidal men, even in the presence of risk factors (e.g., financial problems, mental health issues) and among those making preparations (e.g., attaining means) for suicide. ${ }^{11,17-26}$ Patients generally report openness to inquiry about suicide thoughts, ${ }^{27}$ yet PCCs inquire rarely and inconsistently and may detect only $10-20 \%$ of suicidal patients. ${ }^{28-}$ ${ }^{30}$ Factors contributing to this "conspiracy of silence" include gender-linked norms, ${ }^{31,32}$ stigma, ${ }^{33}$ the belief that PCCs are not equipped to handle mental health issues, ${ }^{34}$ fear of psychiatric hospitalization, ${ }^{35}$ and competing demands during visits. ${ }^{36}$ While interventions targeted to PCCs increased the detection of suicidal patients, many still went unrecognized. ${ }^{29,37}$ 
No interventions have sought to address reticence to disclose suicide thoughts by activating men to disclose or signal receptiveness to discussing their thoughts with a clinician, thereby prompting clinician inquiry. To fill this gap, we developed the Men and Providers Preventing Suicide (MAPS) computer program. The focus of MAPS was to activate middle-aged men with suicide thoughts to discuss the thoughts with their PCC, by providing men a tailored multimedia computer program addressing user-endorsed barriers to discussion. We employed a similar approach in designing MAPS to one we used in developing an earlier depression-focused program, which increased PCC-patient discussion of suicide despite minimal suicide-specific content. ${ }^{38,39}$ Apart from having greater suicide-related content, MAPS also differed from the prior program in being focused specifically on activating middle-aged men regardless of their depression status, since not all who die by suicide are depressed. ${ }^{40}$

In a randomized controlled trial (RCT), we examined the effect of MAPS on discussion of suicide during PCC visits by middle-aged men with recent active suicide thoughts. We hypothesized that when used pre-visit, MAPS would be more effective than an active non-tailored control in promoting suicide discussion. In post hoc exploratory analyses, we also explored moderation of the program's effects by the presence of suicide preparatory behaviors, a risk marker for suicide. ${ }^{41}$, 42

\section{METHODS}

Study activities were conducted from December 2016 through July 2019. The University of California Davis Institutional Review Board provided ethical approval for the study. The RCT was registered in ClinicalTrials.gov (identifier NCT02986113).

\section{Study Setting, Recruitment, and Randomization}

The unit of randomization was the PCC. Patient-level randomization would have entailed greater risk of control arm contamination, since PCCs then would likely have encountered patients from both arms, and exposure to MAPS patients might alter interactions with controls. Participating clinicians were recruited from eight primary care offices in one university-affiliated network in the Sacramento (California) area, during presentations at monthly meetings. The PCCs were told that the trial was comparing two office-based previsit interventions for preventing suicide among middle-aged men but given no details regarding the interventions or the trial outcomes. Enrolled PCCs were randomly assigned to the MAPS or control arm in blinded fashion, using a customcreated computer program that implemented randomization in blocks of varying sizes $(4$ or 8$) .^{43}$

Following randomization, PCCs in both arms were offered four online video modules (30 min total length) summarizing a patient-centered approach to suicide risk assessment. ${ }^{44} \mathrm{We}$ offered this training in response to concerns from stakeholders in pre-RCT interviews that many PCCs lack appropriate training and skills in this realm. ${ }^{35}$ Of the 42 PCCs participating in the trial, eighteen (43\%) viewed at least some of the video content (mean percentage of content viewed 88\% [SD $18.9 \%]$ ), with no significant differences between trial arms in viewing rates or percentage of content viewed.

For patient enrollment, we used reports generated by the health system to identify men aged 35-74 who were assigned to the panel of a participating PCC, regardless of whether they had ever visited the PCC or other clinicians in their office. We telephoned and sent letters to solicit the men's participation and, for those who engaged, conducted telephonic eligibility screening. We offered enrollment to men who were able to read and speak English; self-reported adequate vision, hearing, and hand function to use an interactive computer program on a touchscreen tablet device; had an appointment with their PCC within 2 weeks (or if not, were willing to schedule an appointment in this time frame); and answered "yes" to the question, "In the last four weeks, have you had any (even brief) thoughts of killing yourself?" Men failing to meet any of the foregoing criteria were excluded from participation. Also excluded were men with any of the following: reported or apparent highly unstable medical or mental health status, presence of terminal illness with death anticipated within 3 months, or plan to transfer care from current primary care office within 3 months.

Eligible men agreeing to participate were asked to arrive in their primary care office $1 \mathrm{~h}$ before their visit to complete informed consent and use their assigned intervention. Research assistants logged the men into the study software on touchscreen tablets and showed them how to navigate. The men first completed a pre-intervention questionnaire, which again asked if they had had thoughts of killing themselves in past 4 weeks. We re-administered this item because suicide thoughts may have stopped since the time of eligibility screening for some men, reducing their potential to benefit from trial participation. Thus, men answering "no" to this item were thanked for their time and excluded from further participation.

Following the pre-visit questionnaire, the study software presented each man with the intervention appropriate to his PCC's random group assignment, based on his unique login number. After viewing the intervention, the man attended his PCC visit and then returned to the tablet to complete a postvisit questionnaire. Men completing the post-visit questionnaire were offered a \$20 gift card to a popular retailer.

\section{Study Interventions}

The tailored MAPS program was developed using standard software engineering principles. ${ }^{45}$ Tailored health messages are better remembered, more frequently read, and perceived as more relevant than non-tailored messages. ${ }^{46}$ They are also superior to non-tailored interventions in improving various health behaviors and outcomes across patient populations and target conditions. ${ }^{47}$ The initial version of MAPS was 
created drawing on theory and research relevant to patient activation, ${ }^{48,49}$ suicide behavior, ${ }^{11,17-26}$ and male help seeking for psychological distress. ${ }^{31,32,34,35}$ We included elements prior to work and suggested these would be likely to enhance men's knowledge, skills, and confidence around disclosure of suicide thoughts, leading to greater disclosure of and PCC inquiry regarding suicide thoughts (Fig. 1)..$^{38,39,47,50,51}$ The final version was created after incorporating feedback from pre-RCT interviews with stakeholders in male suicide prevention. ${ }^{35}$ We designed MAPS to have a 15-20-min usage time, commensurate with the typical in-office wait time for a PCC visit. $^{52}$

The computerized content tailoring algorithm was developed using a previously described approach. ${ }^{46}$ The general structure involved sequential empathic acknowledgement of and encouragement to discuss suicide thoughts with the PCC (Fig. 2). The key motivational elements were three "step" modules addressing the following: (1) why and how to talk with a PCC about suicide thoughts, (2) how to negotiate an individualized care plan with the PCC and their team to reduce suicide thoughts, and (3) how to monitor the impact of the plan and work with the care team to modify it when indicated (Fig. 2, box E4a). Consistent with adult learning and behavioral theory, ${ }^{53,54}$ each module allowed some user control over how much information to view by offering optional material. Examples of MAPS content are available from the authors.] $\rightarrow$

Men assigned to the active non-tailored control condition (Fig. 2, box C5) viewed a 3-min sleep hygiene informational video, ${ }^{55}$ followed by a text screen providing encouragement to discuss suicide thoughts with a PCC plus general information about suicide risk factors and support resources. The total usage time for the control condition was approximately $5 \mathrm{~min}$.

\section{Measures}

The primary outcome measure was a single item administered post-visit, asking whether the topic of suicide was discussed during the visit (any vs. no discussion).

We administered an array of other measures pre-visit; where relevant, the number of items and scoring for measures are presented in footnote in Table 1. The Beck Scale for Suicide Ideation (BSSI) was administered to characterize the nature of the suicide thoughts and to assess factors heightening (e.g., strong desire to die) or mitigating (e.g., concerns for family) suicide risk. ${ }^{42,56}$ Four of the BSSI items constituted a Suicide Preparatory Behaviors scale, assessing whether the man had made plans or preparations for suicide, obtained access to means, or made arrangements for what would happen after suicide. Present intent for suicide was measured with a single item. $^{57}$

Additional pre-visit measures concerned health-related issues associated with suicide risk. ${ }^{11,17-26}$ These included a count of eight mental health conditions (eating disorder, depression, anxiety disorder, bipolar disorder, post-traumatic stress disorder, psychosis, childhood sexual abuse, or alcohol or drug problem); the Patient Health Questionnaire (PHQ-9), validated for detecting clinical depression; ${ }^{58}$ the Primary Care

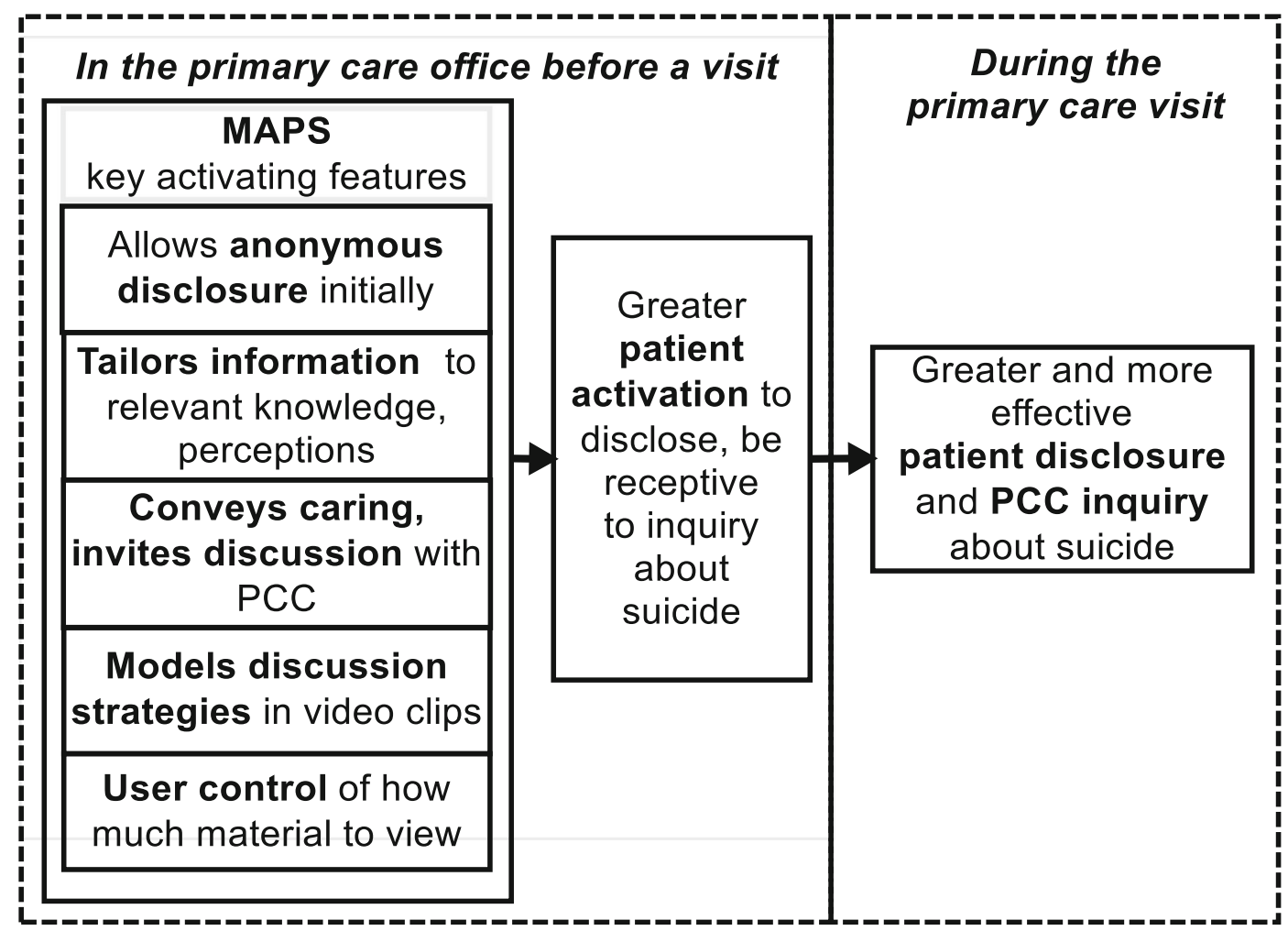

Fig. 1 Conceptual model guiding the development and deployment of MAPS and its intended effects during linked primary care visits MAPS, Men and Providers Preventing Suicide; PCC, primary care clinician. 


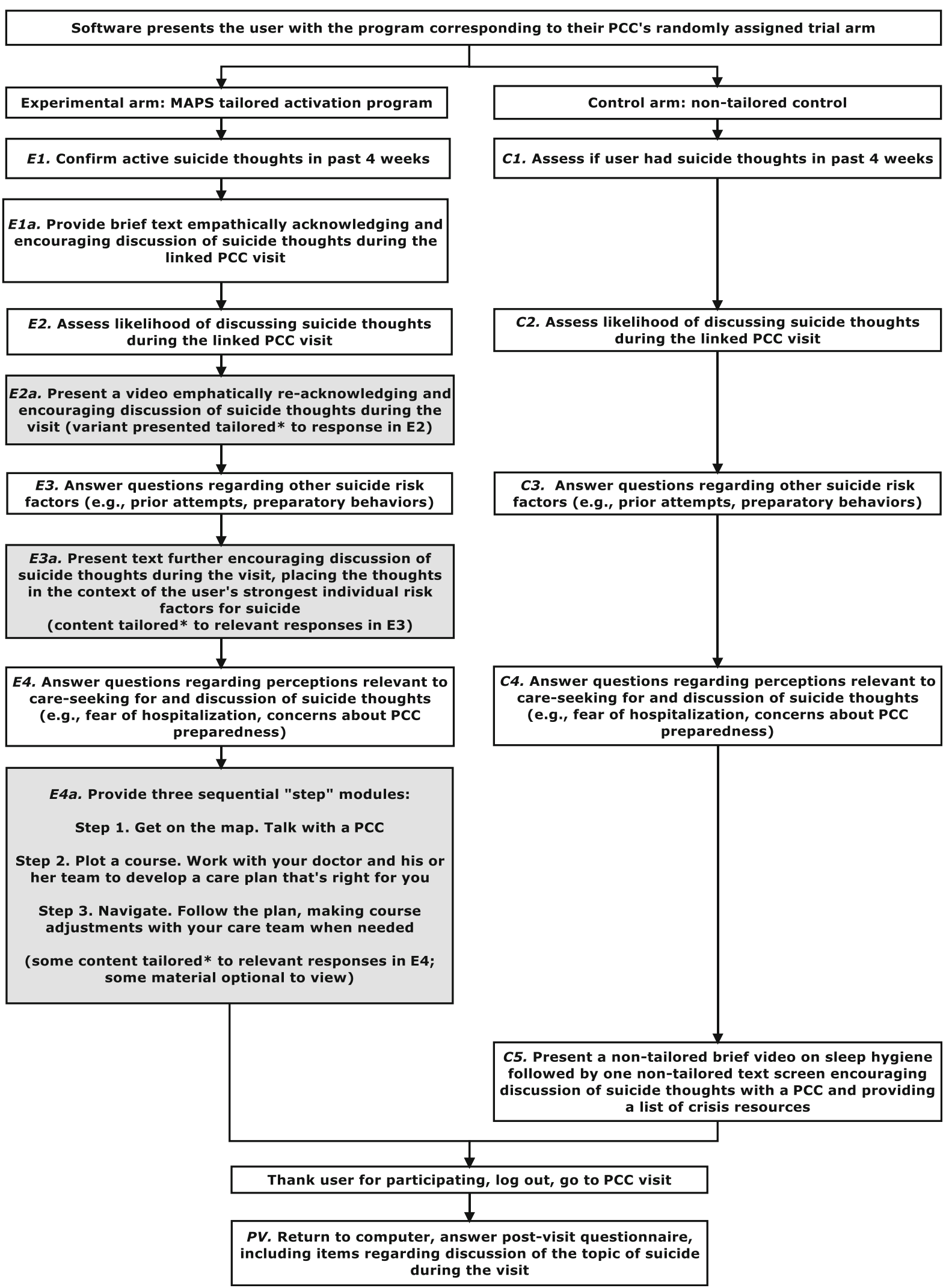

Fig. 2 Overview of the content and sequence of MAPS-tailored activation program and non-tailored control program MAPS, Men and Providers Preventing Suicide; PCC, primary care clinician. The basic structure of tailoring in each module (1) give all users brief feedback tailored to their response(s) to relevant question(s) and (2) offer the option to view more detailed information. 
Table 1 Characteristics of the Study Sample

\begin{tabular}{|c|c|c|c|}
\hline Characteristic & $\begin{array}{l}\text { Active non-tailored } \\
\text { control arm }(N=27)\end{array}$ & $\begin{array}{l}\text { MAPS (experimental) } \\
\operatorname{arm}(N=21)\end{array}$ & $P$ value \\
\hline Age, years, mean (SD) & $55(10)$ & $56(13)$ & 0.82 \\
\hline \multicolumn{4}{|l|}{ Race/ethnicity, no. (\%) } \\
\hline Hispanic (any race) & $5(19)$ & $1(5)$ & \multirow{4}{*}{0.23} \\
\hline Non-Hispanic White & $19(70)$ & $18(86)$ & \\
\hline Non-Hispanic Black & $2(7)$ & $0(0)$ & \\
\hline Non-Hispanic Other & $1(4)$ & $2(10)$ & \\
\hline \multicolumn{4}{|l|}{ Education level, no. (\%) } \\
\hline High-school graduate or less & $1(4)$ & $0(0)$ & \multirow[t]{4}{*}{0.81} \\
\hline Some college & $10(37)$ & $8(38)$ & \\
\hline College graduate & $11(41)$ & $8(38)$ & \\
\hline Any graduate courses & $5(19)$ & $5(24)$ & \\
\hline \multicolumn{4}{|l|}{ Annual household income, no. (\%) } \\
\hline$<\$ 20,000$ & $2(7)$ & $0(0)$ & \multirow[t]{6}{*}{0.44} \\
\hline$\$ 20,000-\$ 34,999$ & $2(7)$ & $2(10)$ & \\
\hline$\$ 35,000-\$ 74,999$ & $7(26)$ & $6(29)$ & \\
\hline$\$ 75,000-\$ 124,999$ & $4(15)$ & $6(29)$ & \\
\hline$\geq \$ 125,000$ & $12(44)$ & $6(29)$ & \\
\hline Decline to answer & $0(0)$ & $1(5)$ & \\
\hline \multicolumn{4}{|l|}{ Sexual orientation, no. (\%) } \\
\hline Heterosexual & $26(96)$ & $16(76)$ & \multirow{3}{*}{0.10} \\
\hline Gay & $1(4)$ & $3(14)$ & \\
\hline Other & $0(0)$ & $2(10)$ & \\
\hline \multicolumn{4}{|l|}{ Marital status, no. (\%) } \\
\hline Married & $20(74)$ & $14(67)$ & \multirow[t]{5}{*}{0.83} \\
\hline Widowed & $1(4)$ & $1(5)$ & \\
\hline Divorced & $2(7)$ & $2(10)$ & \\
\hline Separated & $1(4)$ & $0(0)$ & \\
\hline Never married & 3 (11) & $4(19)$ & \\
\hline Living alone, no. (\%) & $5(19)$ & $5(24)$ & 0.65 \\
\hline Practice any religion/faith, no. (\%) & $6(22)$ & $6(29)$ & 0.61 \\
\hline Toughness score, standardized, mean (SD) ${ }^{\mathrm{a}}$ & $0.1(0.5)$ & $-0.2(0.8)$ & 0.12 \\
\hline \multicolumn{4}{|l|}{ Self-rated health, no. $(\%)$} \\
\hline Excellent & $1(4)$ & $0(0)$ & \multirow[t]{5}{*}{0.38} \\
\hline Very good & $8(30)$ & $2(10)$ & \\
\hline Good & $7(26)$ & $9(43)$ & \\
\hline Fair & $10(37)$ & $9(43)$ & \\
\hline Poor & $1(4)$ & $1(5)$ & \\
\hline No. of medical conditions, mean (SD) ${ }^{b}$ & $3.3(1.4)$ & $3.1(1.9)$ & 0.75 \\
\hline No. of mental health conditions, mean $(\mathrm{SD})^{\mathrm{c}}$ & $2.7(1.7)$ & $3.3(1.9)$ & 0.23 \\
\hline AUDIT-C score, mean (SD) ${ }^{\mathrm{d}}$ & $3.1(2.6)$ & $2.4(2.1)$ & 0.31 \\
\hline \multicolumn{4}{|l|}{ Recreational drug use past year, no. (\%) } \\
\hline Never & $19(70)$ & $10(48)$ & \multirow[t]{5}{*}{0.32} \\
\hline Less than monthly & $4(15)$ & $3(14)$ & \\
\hline Monthly & $0(0)$ & $1(5)$ & \\
\hline Weekly & $0(0)$ & $1(5)$ & \\
\hline Daily or almost daily & $4(15)$ & $6(29)$ & \\
\hline PHQ-9 score, mean (SD) ${ }^{\mathrm{e}}$ & $13.2(7.1)$ & $14.1(6.7)$ & 0.65 \\
\hline PTSD-PC score, mean $(S D)^{f}$ & $1.4(1.3)$ & $1.5(1.4)$ & 0.84 \\
\hline \multicolumn{4}{|l|}{ BSSI scores, mean (SD) } \\
\hline Total $^{\mathrm{g}}$ & $11.5(6.7)$ & $9.7(6.9)$ & 0.37 \\
\hline Suicide Preparatory Behaviors scale ${ }^{\mathrm{h}}$ & $2.0(1.7)$ & $2.1(2.1)$ & 0.79 \\
\hline Suicide intent now, mean $(\mathrm{SD})^{\mathrm{i}}$ & $0.6(1.0)$ & $0.9(1.5)$ & 0.48 \\
\hline
\end{tabular}

${ }^{a}$ Seventeen-item scale, possible score range of 17-85, higher scores $=$ higher gender-linked toughness self-perceptions

${ }^{b}$ From a count of 11 conditions: arthritis/rheumatism, chronic or recurring painful condition, hearing or vision problem, neurological problem, hypertension, heart problem, human immunodeficiency virus infection/acquired immunodeficiency syndrome, diabetes, lung problem, cancer, or sleep problem

${ }^{c}$ From a count of 8 conditions: eating disorder, depression, anxiety disorder, bipolar disorder, post-traumatic stress disorder, psychosis, childhood sexual abuse, alcohol, or drug problem

${ }^{d}$ Three items, possible score range of $0-12$, scores of $>4$ considered optimal for identifying hazardous drinking or active alcohol use disorders in men ${ }^{e}$ Nine items, possible score range of 0-27; scores of 10 or greater are suggestive of clinical depression

${ }^{f}$ Four items, possible score range of $0-4$; scores of $>2$ are suggestive of post-traumatic stress disorder

${ }^{g}$ Nineteen items, possible score range of $0-38$; higher scores are suggestive of higher risk for suicide

${ }^{h}$ Four items, possible score range of 0-8; higher scores are suggestive of higher risk for suicide

${ }^{i}$ Single item, 11-point response scale, 0 (low suicide intent now) to 10 (high suicide intent now)

Posttraumatic Stress Disorder screen, ${ }^{59}$ the Alcohol Use Disorders Identification Test (AUDIT-C) for detecting hazardous drinking and alcohol use disorders; ${ }^{60}$ a single item assessing recreational drug use; a single self-rated health item; and a count of 11 medical conditions (arthritis, chronic, or recurring painful condition, hearing or vision problem, neurological problem, hypertension, heart problem, human immunodeficiency virus infection, diabetes, lung problem, cancer, or sleep 


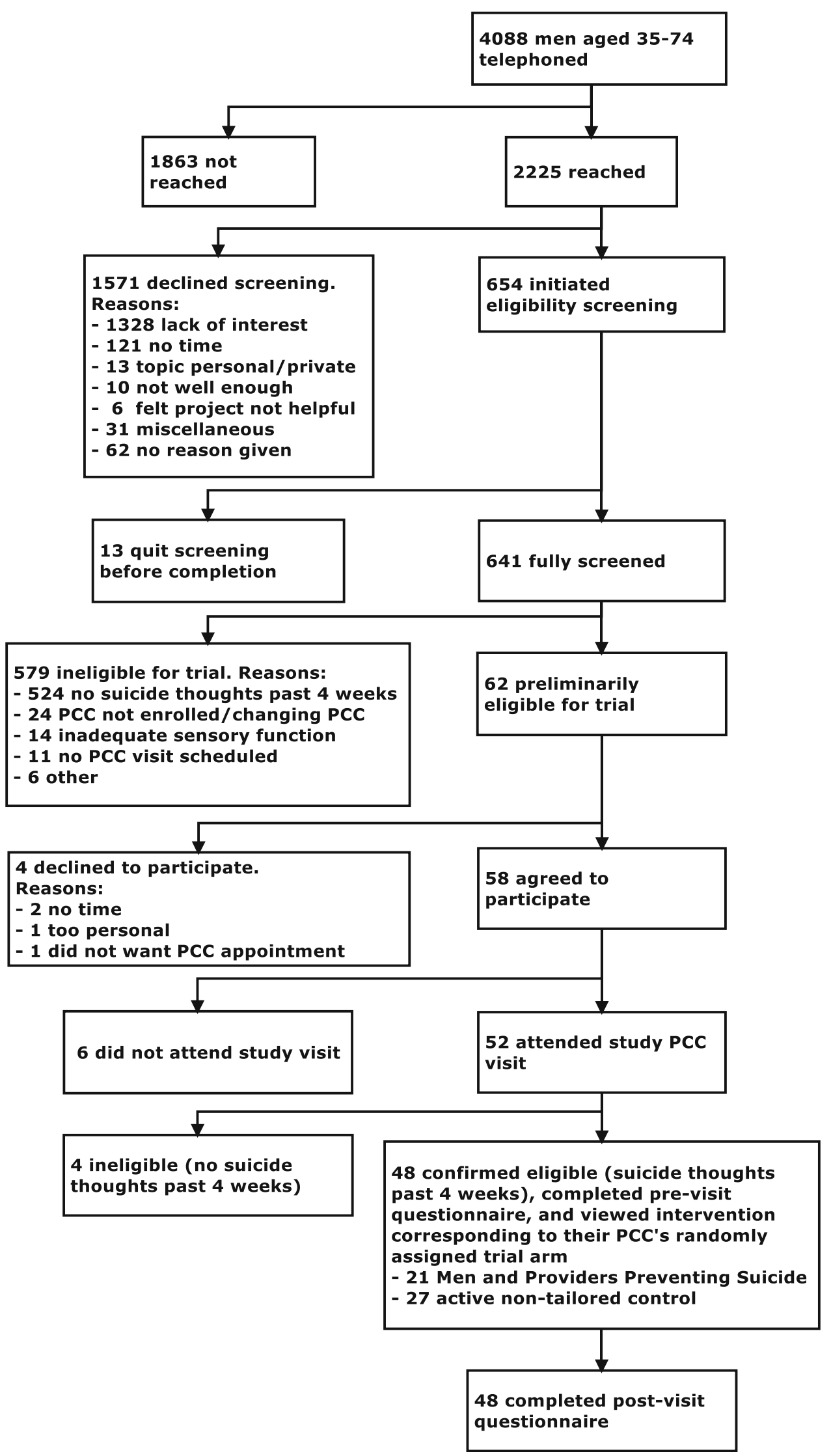

Fig. 3 Flow of participants through the trial. PCC, primary care clinician. 
problem). Sociodemographic measures included age, race/ethnicity, education level, annual household income, sexual orientation, marital/partner status, whether the man was living alone, and whether they practiced any religion or faith.

\section{Data Analysis}

Data were analyzed using Stata 15.1 (Stata Corporation, College Station, TX). Descriptive and inferential statistics (means, standard deviations, percentages, chi-square, or $t$ tests) were used to describe the baseline characteristics of the patient study sample by patient-assigned study group (MAPS or control). A generalized estimating equation (GEE) approach was used to adjust for the nesting of patients within physicians, implemented as logistic regression analyses using binomial distributions, logit links, exchangeable working correlations, and robust standard error estimators. GEE logistic regression analyses examined the relationship between suicide discussion (any vs. none) during the study visit and trial arm.

Another set of GEE logistic regression analyses examined moderation of the effect of MAPS on discussion of suicide during the visit by the presence or absence of suicide preparatory behaviors (assessed with the BSSI). In one of these analyses, stratified by presence or absence of preparatory behaviors, independent (rather than exchangeable) working correlation was used; this was necessary to attain model convergence.

To facilitate interpretation, key regression findings are also reported as adjusted mean marginal effects of intervention, hereafter referred to as nesting-adjusted predicted effects.

\section{RESULTS}

Forty-two PCCs (all physicians) were enrolled and randomized, $20(48 \%)$ to the MAPS arm and $22(52 \%)$ to the active non-tailored control arm. Thirty-two (76\%) PCCs completed a baseline questionnaire: $15(75 \%)$ in the MAPS arm and 17 (77\%) in the control arm. Of the 32 PCCs completing the baseline questionnaire, 21 (65\%) were family physicians and $11(35 \%)$ were general internists, they had practiced on average for 8 years (range 1-22), their mean age was 44 (range 2961), $21(65 \%)$ were female, and 19 (59\%) were non-Hispanic White, 7 (22\%) non-Hispanic Other race, and 5 (26\%) Hispanic (any race). There were no significant differences in PCC characteristics between trial arms.

Figure 3 provides the CONSORT diagram depicting the flow of patients from recruitment through the study activities. Of the 42 PCCs randomized, 16 had no patients enroll in the trial. From the panels of the remaining 26 PCCs, 48 middleaged male patients enrolled (MAPS 21, control 27), a mean of 1.8 (range 1-5) per PCC. Table 1 shows the characteristics of the patients by trial arm; there were no statistically significant differences in characteristics between arms.

Any suicide discussion was more likely among MAPS patients $(15 / 21[65 \%])$ than controls $(8 / 27$ [35\%]). In the nesting adjusted logistic regression, the odds ratio (OR) for an intervention effect on discussion of suicide during the study PCC visit was 5.91 (95\% confidence interval (CI) 1.59-21.94; $P=0.008$ ), a nesting-adjusted predicted effect of $71 \%$ for MAPS and 30\% for control.

In the examination of moderation of the intervention effect by the presence or absence of any suicide preparatory behaviors, the interaction effect was not statistically significant $(P=$ 0.067). Stratified analyses explored the interaction further. Among the 17 patients (8 MAPS and 9 control) without preparatory behaviors, discussion of suicide was reported by $3(37.5 \%)$ of the MAPS patients and $2(22 \%)$ of the controls. This corresponded to an OR of $2.10(95 \%$ CI $0.27-16.59 ; P=$ 0.48 ) (analysis conducted using an independent working correlation). By contrast, among the 31 patients (13 MAPS and 18 control) with suicide preparatory behaviors, suicide discussion was reported by $12(92 \%)$ of the MAPS patients and 6 $(33 \%)$ of the controls. This difference corresponded to an OR of 27.45 (95\% CI 2.74-274.96; $P=0.005$ ), a nesting-adjusted predicted effect of $93 \%$ for MAPS patients and $34 \%$ for control patients.

\section{DISCUSSION}

To our knowledge, this was the first RCT of a primary care suicide prevention intervention targeted to middle-aged men at risk of suicide, regardless of their depression status. ${ }^{1,2}$ The tailored MAPS program was designed to encourage men with active suicide thoughts to discuss the topic with their PCCs, opening the door to treatment to reduce suffering and suicide risk. We found that compared with men viewing an active nontailored control, men using MAPS were significantly more likely to discuss the topic of suicide during a linked PCC visit, a clinically meaningful effect based on the nesting-adjusted predicted effects of $71 \%$ for MAPS versus $30 \%$ for control.

In exploratory post hoc analyses, the effect of MAPS on discussion of suicide appeared to be driven primarily by its impact on men reporting any $(\geq 1)$ suicide preparatory behaviors, such as making plans or attaining means. This finding must be interpreted cautiously, given the preliminary and post hoc nature of the analysis and our small sample size. Nonetheless, prior work indicates preparatory behaviors are a marker of heightened risk for suicide. ${ }^{41,42}$ Of note, recruiting the 48 patients in our trial required making phone calls to several thousand men (Fig. 3). Clearly, harnessing the full potential of MAPS in routine practice will require more efficient ways of targeting men most likely to act on suicide thoughts, such as those with preparatory behaviors. One promising approach may be to utilize patient-facing electronic health record portals to offer screening for suicide thoughts and risk factors (including preparatory behaviors) to all middle-aged men in a given health system, followed by access to MAPS (via the portal and/or in primary care offices before visits) when indicated by screening. If this or other efficient targeting approaches can be 
implemented, MAPS may have potential as a time- and resource-efficient tool for activating men with thoughts of suicide and who have undertaken preparatory behaviors to engage with PCCs in discussing the topic. Given that most men who die by suicide have not made a prior suicide attempt, ${ }^{11,12}$ additional RCTs of MAPS that enroll larger samples and stratify enrollment by the presence or absence of preparatory behaviors would be useful.

Despite the positive impact of MAPS, 35\% of men viewing the program reported not discussing suicide during their PCC visit. Some of these men might be "reached" through additional efforts, such as adding more content to MAPS; broadening its scope to leverage supportive relationships with partners, family, or friends; and coupling its use with complimentary community-based and public health interventions. Preliminarily, it was encouraging that of the 13 men viewing MAPS who reported suicide preparatory behaviors, 12 (92\%) reported discussing suicide during the visit. It may be valuable to PCCs to have a focused, high-risk group of men to target for discussion of suicide, given competing demands in office visits. Still, it remains to be studied whether PCC discussion of suicide with such men can help prevent suicide. Future reports from our ongoing RCT will explore this issue.

Our study had limitations. In addition to difficulties with patient recruitment and a resulting small sample size, the patients (and PCCs) were drawn from primary care offices in a single health system in one region of California. It remains uncertain whether the findings generalize to other contexts, such as medical subspecialty care settings or men who are not engaged in healthcare. The experimental MAPS program was longer than the control program (15-20 $\mathrm{min}$ vs. $5 \mathrm{~min}$, respectively). It is possible that the longer length of MAPS contributed to its greater effect on discussion of suicide, independent of its other features (e.g., tailoring of content). We measured suicide discussion during visits using patient report, which is subject to biases. Alternative methods of assessing visit behaviors also have limitations. For example, clinician reports may also be biased, and observing or recording visits may change (e.g., optimize) the behaviors, apart from intervention effects.

In conclusion, in addressing prevalent barriers to discussing suicide, the MAPS-tailored multimedia computer program was successful in activating middle-aged men with thoughts of suicide to engage with their PCCs around this customarily taboo topic. Further, in exploratory post hoc analyses, this effect of MAPS was driven primarily by its salutary impact among men with suicide thoughts who had also undertaken behaviors to prepare for suicide. If these findings are verified in larger, appropriately designed RCTs, MAPS may be an effective, time- and resource-efficient tool for activating men at heightened risk of suicide to engage in potentially life-saving discussion with PCCs.

Acknowledgments: We are indebted to the primary care offices, PCCs, and patients who participated in the trial. We are also grateful to the following individuals: Peach Dounias, BS, Sherry Hao, BA, Lauren Walker, MSW, and Zachary Weiss, BA, who facilitated the patient recruitment and participation; Simon Dvorak, BA, Charles Turner, $\mathrm{PhD}$, and Robert Burnett, MA, who programmed the MAPS and control interventions; the actors who performed in videos incorporated in MAPS, overseen, and trained by Lynn Baker-Nauman, MA; and the analysts at the University of California Davis Clinical and Translational Science Center, for the administrative support in conducting the trial.

Corresponding Author: Anthony Jerant, MD; Department of Family and Community Medicine, University of California Davis (UCD) School of Medicine, 4860 Y Street, Suite 2300, Sacramento, CA 95817, USA (e-mail: afjerant@ucdavis.edu).

Funding Information This work was supported by cooperative agreement U01CE002664 (Jerant, Duberstein) from the National Center for Injury Prevention and Control, U.S. Centers for Disease Control and Prevention (CDC). Additional funding was provided by the UCD Behavioral Health Center of Excellence (Jerant) and the Department of Family and Community Medicine (Jerant). The CDC participated in the design and conduct of the study. Neither the CDC nor the other funders had a role in the collection, management, analysis, or interpretation of the data or in the decision to submit the manuscript for publication.

\section{Compliance with Ethical Standards:}

Conflict of Interest: The authors declare that they do not have a conflict of interest.

Disclaimer: The conclusions and findings in this report are those of the authors and do not necessarily represent the official position of the U.S. Centers for Disease Control and Prevention.

\section{REFERENCES}

1. Suicide. Facts at a glance. Atlanta, GA: National Center for Injury Prevention and Control, Division of Violence Prevention, Centers for Disease Control and Prevention; 2015.

2. Curtin SC, Warner $\mathbf{M}$, Hedegaard $\mathbf{H}$. Increase in suicide in the United States, 1999-2014. NCHS Data Brief. 2016:1-8.

3. 10 leading causes of death by age group, United States - 2017. U.S. Centers for Disease Control and Prevention. 2019; https://www.cdc.gov/ injury/wisqars/leadingcauses.html

4. Shepard DS, Gurewich D, Lwin AK, Reed GA, Jr., Silverman MM. Suicide and Suicidal Attempts in the United States: Costs and Policy Implications. Suicide Life Threat Behav. 2016;46:352-362.

5. Injury Prevention \& Control: Data \& Statistics (WISQARS). U.S. Centers for Disease Control and Prevention. 2016; http://www.cdc.gov/injury/ wisqars/index.html. Accessed 11 March 2020.

6. Ahmedani BK, Simon GE, Stewart C, et al. Health care contacts in the year before suicide death. J Gen Intern Med. 2014;29:870-877.

7. Brown GK, Ten Have T, Henriques GR, Xie SX, Hollander JE, Beck AT. Cognitive therapy for the prevention of suicide attempts: a randomized controlled trial. JAMA. 2005;294:563-570.

8. Linehan MM, Comtois KA, Murray AM, et al. Two-year randomized controlled trial and follow-up of dialectical behavior therapy vs therapy by experts for suicidal behaviors and borderline personality disorder. Arch Gen Psychiatry. 2006;63:757-766.

9. Motto JA, Bostrom AG. A randomized controlled trial of postcrisis suicide prevention. Psychiatr Serv. 2001:52:828-833.

10. du Roscoat E, Beck F. Efficient interventions on suicide prevention: a literature review. Rev Epidemiol Sante Publique. 2013;61:363-374.

11. Kessler RC, Borges G, Walters EE. Prevalence of and risk factors for lifetime suicide attempts in the National Comorbidity Survey. Arch Gen Psychiatry. 1999;56:617-626.

12. Mann JJ. A current perspective of suicide and attempted suicide. Ann Intern Med. 2002;136:302-311.

13. Yip PS, Caine E, Yousuf S, Chang SS, Wu KC, Chen YY. Means restriction for suicide prevention. Lancet. 2012;379:2393-2399.

14. Simon RI. Suicide risk assessment: gateway to treatment and management. In: Simon RI, Hales RE, eds. The American Psychiatric Publishing 
textbook of suicide assessment and management, 2nd Washington, DC: American Psychiatric Publishing; 2012.

15. Clinical Care and Intervention Task Force, National Action Alliance for Suicide Prevention. Suicide care in systems framework. Washington, DC: NAASP; 2011

16. Office of the Surgeon General; National Action Alliance for Suicide Prevention. 2012 national strategy for suicide prevention: goals and objectives for action. Washington, DC: U.S. Department of Health and Human Services; 2012.

17. ten Have $\mathbf{M}$, de Graaf $\mathbf{R}$, van Dorsselaer $\mathbf{S}$, et al. Incidence and course of suicidal ideation and suicide attempts in the general population. Can $J$ Psychiatry. 2009;54:824-833.

18. Cavanagh JT, Carson AJ, Sharpe M, Lawrie SM. Psychological autopsy studies of suicide: a systematic review. Psychol Med. 2003;33:395-405.

19. McDowell AK, Lineberry TW, Bostwick JM. Practical suicide-risk management for the busy primary care physician. Mayo Clin Proc. 2011;86:792-800.

20. Hawton K, Houston K, Haw C, Townsend E, Harriss L. Comorbidity of axis I and axis II disorders in patients who attempted suicide. Am J Psychiatry. 2003;160:1494-1500.

21. Milner A, Sveticic J, De Leo D. Suicide in the absence of mental disorder? A review of psychological autopsy studies across countries. Int $J$ Soc Psychiatry. 2013;59:545-554.

22. Heikkinen M, Aro H, Lonnqvist J. Recent life events, social support and suicide. Acta Psychiatr Scand Suppl. 1994;377:65-72.

23. Olfson M, Weissman MM, Leon AC, Sheehan DV, Farber L. Suicidal ideation in primary care. J Gen Intern Med. 1996;11:447-453.

24. Rudd MD, Berman AL, Joiner TE, Jr., et al. Warning signs for suicide: theory, research, and clinical applications. Suicide Life Threat Behav. 2006;36:255-262.

25. Conner KR, Duberstein PR, Conwell Y, Seidlitz L, Caine ED. Psychological vulnerability to completed suicide: a review of empirical studies. Suicide Life Threat Behav. 2001;31:367-385.

26. Nock MK, Borges G, Bromet EJ, et al. Cross-national prevalence and risk factors for suicidal ideation, plans and attempts. $\mathrm{Br} J$ Psychiatry. 2008; 192:98-105

27. Zimmerman M, Lish JD, Lush DT, Farber NJ, Plescia G, Kuzma MA Suicidal ideation among urban medical outpatients. $J$ Gen Intern Med. 1995; 10:573-576.

28. Feldman MD, Franks P, Duberstein PR, Vannoy S, Epstein R, Kravitz RL. Let's not talk about it: suicide inquiry in primary care. Ann Fam Med. 2007;5:412-418.

29. Nutting PA, Dickinson LM, Rubenstein LV, Keeley RD, Smith JL Elliott CE. Improving detection of suicidal ideation among depressed patients in primary care. Ann Fam Med. 2005;3:529-536.

30. Vannoy SD, Robins LS. Suicide-related discussions with depressed primary care patients in the USA: gender and quality gaps. A mixed methods analysis. BMJ Open. 2011;1:e000198.

31. O'Loughlin RE, Duberstein PR, Veazie PJ, et al. Role of the genderlinked norm of toughness in the decision to engage in treatment for depression. Psychiatr Serv. 2011;62:740-746.

32. Rochlen AB, Paterniti DA, Epstein RM, Duberstein P, Willeford L Kravitz RL. Barriers in diagnosing and treating men with depression: a focus group report. Am J Mens Health. 2010;4:167-175.

33. Reynders A, Kerkhof AJ, Molenberghs G, Van Audenhove C. Helpseeking, stigma and attitudes of people with and without a suicidal past. A comparison between a low and a high suicide rate country. $J$ Affect Disord. 2015; 178:5-11.

34. Kravitz RL, Paterniti DA, Epstein RM, et al. Relational barriers to depression help-seeking in primary care. Patient Educ Couns. 2011;82:207-213

35. Jerant A, Duberstein P, Cipri C, Bullard B, Stone D, Paterniti D. Stakeholder views regarding a planned primary care office-based interactive multimedia suicide prevention tool. Patient Educ Couns. 2019; 102:332-339

36. Rost K, Nutting P, Smith J, Coyne JC, Cooper-Patrick L, Rubenstein L. The role of competing demands in the treatment provided primary care patients with major depression. Arch Fam Med. 2000;9:150-154.

37. Almeida OP, Pirkis J, Kerse N, et al. A randomized trial to reduce the prevalence of depression and self-harm behavior in older primary care patients. Ann Fam Med. 2012;10:347-356.

38. Shah R, Franks P, Jerant A, et al. The effect of targeted and tailored patient depression engagement interventions on patient-physician discussion of suicidal thoughts: a randomized control trial. J Gen Intern Med. 2014;29:1148-1154.

39. Kravitz RL, Franks P, Feldman MD, et al. Patient engagement programs for recognition and initial treatment of depression in primary care: a randomized trial JAMA. 2013;310:1818-1828.

40. Nock MK, Hwang I, Sampson NA, Kessler RC. Mental disorders, comorbidity and suicidal behavior: results from the National Comorbidity Survey Replication. Mol Psychiatry. 2010;15:868-876.

41. Hunt T, Wilson CJ, Caputi P, Woodward A, Wilson I. Signs of current suicidality in men: A systematic review. PLoS One. 2017;12:e0174675.

42. Beck AT, Brown GK, Steer RA, Dahlsgaard KK, Grisham JR. Suicide ideation at its worst point: a predictor of eventual suicide in psychiatric outpatients. Suicide Life Threat Behav. 1999;29:1-9.

43. Schulz KF, Grimes DA. Generation of allocation sequences in randomised trials: chance, not choice. Lancet. 2002;359:515-519.

44. Pisani AR, Cross WF, Watts A, Conner K. Evaluation of the Commitment to Living (CTL) curriculum: a 3-hour training for mental health professionals to address suicide risk. Crisis. 2012;33:30-38.

45. Pressman RS. Software Engineering: A Practitioner's Approach, 6th New York: McGraw-Hill; 2005.

46. Kreuter M, Farrell D, Olevitch L, Brennan L. Tailoring Health Messages: Customizing Communication With Computer Technology. Mahwah: Lawrence Erlbaum Associates; 2000.

47. Noar SM, Benac CN, Harris MS. Does tailoring matter? Meta-analytic review of tailored print health behavior change interventions. Psychol Bull. 2007;133:673-693.

48. Hibbard JH, Greene $\mathbf{J}$. What the evidence shows about patient activation: better health outcomes and care experiences; fewer data on costs. Health Aff (Millwood). 2013;32:207-214.

49. Sacks RM, Greene J, Hibbard JH, Overton V. How well do patient activation scores predict depression outcomes one year later? $J$ Affect Disord. 2014;169:1-6.

50. Jerant A, To P, Franks P. The Effects of Tailoring Knowledge Acquisition on Colorectal Cancer Screening Self-Efficacy. J Health Commun. 2014;In press.

51. Jerant A, Sohler N, Fiscella K, Franks B, Franks P. Tailored interactive multimedia computer programs to reduce health disparities: opportunities and challenges. Patient Educ Couns. 2011;85:323-330.

52. Medical practice pulse report: patient perspectives on American health care. Press Ganey Associates, Inc. 2009; http://www.pressganey.com/ Documents_secure/Medical\%20Practices/White\%20Papers/Keep_Me_ Waiting.pdf?viewFile. Accessed 11 March 2020.

53. Peterson C, Stunkard AJ. Personal control and health promotion. Soc Sci Med. 1989;28:819-828.

54. Miller SM. Monitoring versus blunting styles of coping with cancer influence the information patients want and need about their disease. Implications for cancer screening and management. Cancer. 1995;76: 167-177.

55. Epstein A. Common Sleeping Problems (video). New York: HealthiNation; 2011.

56. Beck AT, Brown GK, Steer RA. Psychometric characteristics of the Scale for Suicide Ideation with psychiatric outpatients. Behav Res Ther. 1997;35: 1039-1046.

57. Linehan MM, Comtois KA, Brown MZ, Heard HL, Wagner A. Suicide Attempt Self-Injury Interview (SASII): development, reliability, and validity of a scale to assess suicide attempts and intentional self-injury. Psychol Assess. 2006; 18:303-312.

58. Kroenke K, Spitzer RL, Williams JB. The PHQ-9: validity of a brief depression severity measure. J Gen Intern Med. 2001;16:606-613.

59. Prins A, Bovin MJ, Smolenski DJ, et al. The Primary Care PTSD Screen for DSM-5 (PC-PTSD-5): Development and evaluation within a veteran primary care sample. J Gen Intern Med. 2016;31:1206-1211.

60. Bush K, Kivlahan DR, McDonell MB, Fihn SD, Bradley KA. The AUDIT alcohol consumption questions (AUDIT-C): an effective brief screening test for problem drinking. Ambulatory Care Quality Improvement Project (ACQUIP). Alcohol Use Disorders Identification Test. Arch Intern Med. 1998; 158: 1789-1795.

Publisher's Note: Springer Nature remains neutral with regard to jurisdictional claims in published maps and institutional affiliations. 